\title{
Modeling and Bayesian parameter estimation for shape memory alloy bending actuators
}

\author{
John H. Crews ${ }^{a}$ and Ralph C. Smith ${ }^{a}$ \\ ${ }^{a}$ Department of Mathematics, Center for Research in Scientific Computation, North Carolina \\ State University, Raleigh, NC, United States
}

\begin{abstract}
In this paper, we employ a homogenized energy model (HEM) for shape memory alloy (SMA) bending actuators. Additionally, we utilize a Bayesian method for quantifying parameter uncertainty. The system consists of a SMA wire attached to a flexible beam. As the actuator is heated, the beam bends, providing endoscopic motion. The model parameters are fit to experimental data using an ordinary least-squares approach. The uncertainty in the fit model parameters is then quantified using Markov Chain Monte Carlo (MCMC) methods. The MCMC algorithm provides bounds on the parameters, which will ultimately be used in robust control algorithms. One purpose of the paper is to test the feasibility of the Random Walk Metropolis algorithm, the MCMC method used here.
\end{abstract}

Keywords: shape memory alloys, uncertainty quantificiation, markov chain monte carlo

\section{INTRODUCTION}

Shape memory alloys (SMA) are unique actuators that are gaining increased utilization in prototypes such as robotic catheters, ${ }^{1,2}$ robotic hands, ${ }^{3}$ jet chevrons, ${ }^{4,5}$ and underwater vessels. ${ }^{6}$ SMA actuators are capable of recovering large strains (approximately 5\%) upon heating. However, the control and optimization of SMA devices is complicated by the material's nonlinear, hysteretic dependence on stress and temperature.

In this paper we employ a computationally efficient model for a SMA bending actuator. Furthermore, we utilize Markov Chain Monte Carlo (MCMC) methods to quantify parameter uncertainty in a systematic manner. MCMC methods are based on Bayes' rule, which relates the posterior density of model parameters to a prior density and the likelihood of those parameters. ${ }^{7,8}$ MCMC methods avoid the difficulty of calculating the likelihoods directly (and integrating over the parameter space) by creating a Markov Chain where the stationary distribution is the posterior distribution. A proposal function and an accept-reject rule determine the random walk of the Markov Chain. ${ }^{9}$ The main goal of this paper is to test the feasibility of the approach for the SMA bending actuator under consideration.

An algorithmic approach to quantifying parametric uncertainty is useful for numerous reasons. The results can be used to produce bounds on the model output for comparison to experimental data. An additional use for SMA actuators (in particular the bending actuator under consideration) is to quantify parameter uncertainty for control algorithms. Robust control methods require some measure of model uncertainty in order to guarantee stability or optimality. For example, sliding mode control (or variable structure control) requires bounds on model uncertainty in order to ensure the attractiveness of the sliding surface. ${ }^{10}$

The remainder of this paper is organized as follows. The model of a flexible beam actuated by a single SMA tendon is presented first. The homogenized energy model (HEM) is used, which has been applied to various smart materials. ${ }^{11-20}$ The approach to quantify uncertainty is described, including optimization of model parameters and the Markov Chain Monte Carlo method used here. Finally, the results are presented.

Further author information: (Send correspondence to J. Crews)

J. Crews: E-mail: jhcrews@ncsu.edu, Telephone: 19195150684 


\section{HOMOGENIZED ENERGY MODEL OF A SINGLE-TENDON SHAPE MEMORY ALLOY BENDING ACTUATOR}

The system consists of a flexible beam actuated by a single shape memory alloy (SMA) tendon, as depicted in Figure 1(a). The SMA tendon (actuator) is held a fixed distance from the neutral axis of the flexible beam using rapid-prototyped collets. The SMA actuator is pre-strained before it is attached to the axially stiff, laterally compliant beam. Therefore, a moment is created as the SMA tendon contracts due to Joule heating and the structure bends in the manner shown in Figure 1(b). Similar robotic systems include catheters and smart inhalers. ${ }^{21,22}$ A mesoscopic free energy model of a catheter was introduced in Veeramani et al., ${ }^{1}$ with further work in Crews et al. ${ }^{2,23}$

\subsection{System Model}

As shown in, ${ }^{2,23}$ the bending angle $\theta(t)$ can be related to the SMA tendon stress $\sigma(t)$ by the relation

$$
\theta(t)=\frac{a A_{c} L \sigma(t)}{E I},
$$

where $a$ is the actuator offset from the neutral axis, $A_{c}$ is the cross-sectional area of the SMA actuator, $L$ is its length, $E$ is the elastic (Young's) modulus of the flexible beam, and $I$ is its area moment of inertia. The stress-strain relationship for the bending system can be simplified to

$$
\sigma(t)=\frac{E I}{a^{2} A_{c}}\left(\varepsilon_{P}-\varepsilon(t)\right)
$$

where $\varepsilon_{P}$ is the pre-strain in the SMA actuator. Equation (2) reduces the planar bending problem to a onedimensional problem consisting of a SMA actuator in parallel with a linear spring with stiffness

$$
K=\frac{E I}{a^{2} A_{c}} .
$$

The SMA strain $\varepsilon(t)$ is modeled using the homogenized energy model. The HEM accounts for material inhomogeneities and interaction effects to accurately and efficiently quantify the macroscopic SMA behavior. A complete derivation of the model and data-driven techniques for estimating model parameters are provided in. ${ }^{17}$ In Crews et al. ${ }^{17}$ a single SMA actuator in constant-temperature conditions was considered. The relevant relationships and equations are summarized here in order to tailor the HEM to the bending system under consideration.

The HEM quantifies the macroscopic strain

$$
\varepsilon(t)=\int_{0}^{\infty} \int_{-\infty}^{\infty} \nu_{R}\left(\sigma_{R}\right) \nu\left(\sigma_{I}\right) \bar{\varepsilon}\left(\sigma(t)+\sigma_{I}, T(t) ; \sigma_{R}\right) d \sigma_{I} d \sigma_{R}
$$

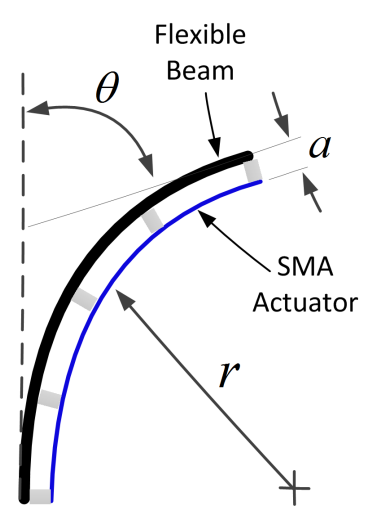

(a)

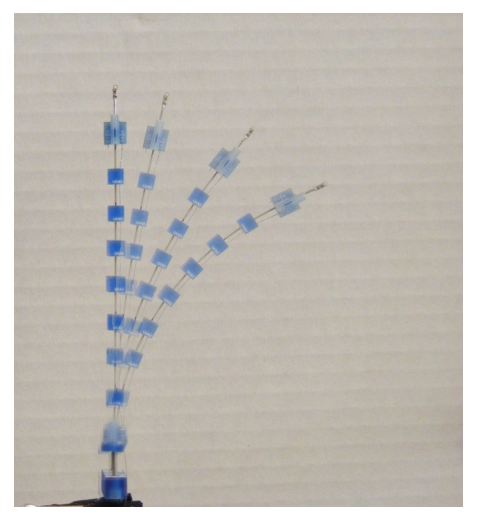

(b)

Figure 1: (a) Flexible beam actuated by single SMA tendon; (b) Time-lapse photograph of robotic system. 
by assuming the relative stress $\sigma_{R}$ and interaction stress $\sigma_{I}$ are manifestations of underlying densities $\nu_{R}\left(\sigma_{R}\right)$ and $\nu_{I}\left(\sigma_{I}\right)$. In Equation (4), $T(t)$ is the temperature in the SMA wire. Different forms can be assumed for the densities, subject to certain conditions. ${ }^{11}$ Here, we assume the densities

$$
\nu_{R}\left(\sigma_{R}\right)=\frac{1}{C_{1}} \sum_{m=1}^{M_{\alpha}} \sum_{k=1}^{K_{\alpha}} \alpha_{k, m} \phi_{k, m}\left(\sigma_{R}\right) \quad C_{1}=\sum_{m=1}^{M_{\alpha}} \sum_{k=1}^{K_{\alpha}} \alpha_{k, m}
$$

and

$$
\nu_{I}\left(\sigma_{I}\right)=\frac{1}{C_{2}} \sum_{k=1}^{K_{\beta}} \beta_{k} \psi_{k}\left(\sigma_{I}\right) \quad C_{2}=\sum_{k=1}^{K_{\beta}} \beta_{k}
$$

are linear combinations of log-normal and normal functions. The kernels $\phi_{k, m}\left(\sigma_{R}\right)$ and $\psi_{k}\left(\sigma_{I}\right)$ are given by

$$
\phi_{k, m}\left(\sigma_{R}\right)=\frac{1}{c_{k} \sigma_{R} \sqrt{2 \pi}} \exp \left(-\left[\ln \left(\sigma_{R}\right)-\mu_{R_{m}}\right]^{2} / 2 c_{k}^{2}\right)
$$

and

$$
\psi_{k}\left(\sigma_{I}\right)=\frac{1}{b_{k} \sqrt{2 \pi}} \exp \left(-\sigma_{I}^{2} / 2 b_{k}^{2}\right)
$$

The density coefficients $\alpha_{k, m}$ and $\beta_{k}$ and parameters $c_{k}, \mu_{R_{m}}$, and $b_{k}$ can be estimated using experimental data. ${ }^{17}$

The mesoscopic (or local) strain $\bar{\varepsilon}\left(\sigma(t)+\sigma_{I}, T(t) ; \sigma_{R}\right)$ in Equation (4) is given by

$$
\bar{\varepsilon}\left(\sigma(t)+\sigma_{I}, T(t) ; \sigma_{R}\right)=x_{A}(t) \frac{\sigma(t)}{E_{A}}+x_{M+}(t)\left(\frac{\sigma(t)}{E_{M}}+\varepsilon_{T}\right)+x_{M-}(t)\left(\frac{\sigma(t)}{E_{M}}-\varepsilon_{T}\right),
$$

where $x_{A}(t), x_{M+}(t)$, and $x_{M-}(t)$ are the austenite $(A)$, martensite plus $(M+)$, and martensite minus $(M-)$ phase fractions, respectively, $E_{A}$ is the austenitic elastic modulus, $E_{M}$ is the martensitic elastic modulus, and $\varepsilon_{T}$ is the maximum recoverable strain.

The evolution of the phase fractions is governed by the coupled differential equations

$$
\begin{aligned}
& \dot{x}_{M+}(t)=-p_{+A} x_{M+}(t)+p_{A+} x_{A}(t) \\
& \dot{x}_{M-}(t)=-p_{-A} x_{M-}(t)+p_{A-} x_{A}(t)
\end{aligned}
$$

and the conservation relation

$$
x_{A}(t)=1-x_{M+}(t)-x_{M-}(t) .
$$

Substituting (8) into (7) yields

$$
\begin{aligned}
& \dot{x}_{M+}(t)=-\left(p_{+A}+p_{A+}\right) x_{M+}(t)-p_{A+} x_{M-}(t)+p_{A+} \\
& \dot{x}_{M-}(t)=-\left(p_{-A}+p_{A-}\right) x_{M-}(t)-p_{A-} x_{M+}(t)+p_{A-} .
\end{aligned}
$$

The transition rates $p_{\alpha \beta}(\alpha, \beta=A, M+, M-)$ depend on the SMA stress, temperature, and material parameters and are described in greater detail in Crews et al. ${ }^{17}$ The temperature in the SMA actuator is quantified using a first-order, lumped capacitance model,

$$
\dot{T}(t)=-h\left(T(t)-T_{\infty}\right)+\gamma v^{2}(t),
$$

where $h$ is the convective heat transfer coefficient between the actuator and ambient air at temperature $T_{\infty}, v(t)$ is the applied voltage, and $\gamma$ is a parameter that accounts for the SMA resistance and specific heat.

The integral (4) is discretized using 4-point Gaussian quadrature on 20 equal intervals, yielding

$$
\varepsilon(t)=\sum_{i=1}^{N_{i}} \sum_{j=1}^{N_{j}} \nu_{R}\left(\sigma_{R_{i}}\right) \nu_{I}\left(\sigma_{I_{j}}\right) \bar{\varepsilon}\left(\sigma(t)+\sigma_{I_{j}}, T(t) ; \sigma_{R_{i}}\right) v_{i} w_{j},
$$


where $\sigma_{R_{i}}$ and $\sigma_{I_{j}}$ are the quadrature points and $v_{i}$ and $w_{j}$ are the quadrature weights. The summations in (11) can be converted to the vector-matrix-vector product

$$
\varepsilon(t)=V^{T} \Gamma W,
$$

where

$$
\begin{gathered}
V^{T}=\left[v_{1} \nu_{R}\left(\sigma_{R_{1}}\right), \cdots, v_{N_{i}} \nu_{R}\left(\sigma_{R_{N_{i}}}\right)\right] \\
W^{T}=\left[w_{1} \nu_{I}\left(\sigma_{I_{1}}\right), \cdots, w_{N_{j}} \nu_{I}\left(\sigma_{I_{N_{j}}}\right)\right] \\
\Gamma=X_{A} \frac{\sigma(t)}{E_{A}}+X_{M+}\left(\frac{\sigma(t)}{E_{M}}+\varepsilon_{T}\right)+X_{M-}\left(\frac{\sigma(t)}{E_{M}}-\varepsilon_{T}\right) .
\end{gathered}
$$

The $N_{i} \times N_{j}$ matrices $X_{A}, X_{M+}$, and $X_{M-}$ evaluate the phase fractions at

$$
\left[X_{\alpha}(t, \sigma(t), T(t))\right]_{i j}=x_{\alpha}\left(t, \sigma(t)+\sigma_{I_{j}}, T(t) ; \sigma_{R_{i}}\right) \quad \alpha=A, M+, M-,
$$

where $x_{\alpha}$ are the solutions to (7) and (8).

The ODEs (9) are discretized and solved using an implicit Euler scheme. For discretized time $t^{k}=k \Delta t$, stress $\sigma^{k}=\sigma\left(t^{k}\right)$, and temperature $T^{k}=T\left(t^{k}\right)$ values, the implicit Euler discretization yields

$$
\begin{aligned}
& a_{11}^{k+1} x_{M+}^{k+1}+a_{12}^{k+1} x_{M-}^{k+1}=x_{M+}^{k}+a_{13}^{k+1} \\
& a_{21}^{k+1} x_{M+}^{k+1}+a_{22}^{k+1} x_{M-}^{k+1}=x_{M-}^{k}+a_{23}^{k+1},
\end{aligned}
$$

where

$$
\begin{array}{rlrl}
a_{11}^{k+1} & =1+\Delta t\left(p_{+A}+p_{A+}\right) & & a_{21}^{k+1}=p_{A-} \Delta t \\
a_{12}^{k+1} & =p_{A+} \Delta t & & a_{22}^{k+1}=1+\Delta t\left(p_{-A}+p_{A-}\right) \\
a_{13}^{k+1}=p_{A+} \Delta t & a_{23}^{k+1}=p_{A-} \Delta t .
\end{array}
$$

Note that in (13), the transition rates depend on $\sigma^{k}, T^{k}, \sigma_{I}$, and $\sigma_{R}$. The austentic phase fraction $x_{A}^{k+1}$ is given by the conservation relation (8). The implicit Euler discretization of the temperature yields

$$
T^{k+1}=d_{1}^{k+1} T^{k}+d_{2}^{k+1},
$$

where

$$
d_{1}^{k+1}=\frac{1}{1+h \Delta t} \quad d_{2}^{k+1}=\frac{\Delta t\left(h T_{\infty}+\gamma\left(v^{k+1}\right)^{2}\right)}{1+h \Delta t} .
$$

The stress $\sigma^{k}$ is found by substituting (11) into (2) and solving for the equilibrium stress, which yields

$$
\sigma^{k}=\frac{\varepsilon_{P}-\varepsilon_{T}\left(V^{T} X_{M+} W-V^{T} X_{M-} W\right)}{\frac{a^{2} A_{c}}{E I}+V^{T} X_{A} W \frac{1}{E_{A}}+V^{T} X_{M+} W \frac{1}{E_{M}}+V^{T} X_{M-} W \frac{1}{E_{M}}} .
$$

Equation (12) can be solved using Cramer's rule, which gives

$$
\begin{aligned}
& x_{M+}^{k+1}=c_{11}^{k+1} x_{M+}^{k}+c_{12}^{k+1} x_{M-}^{k}+c_{13}^{k+1} \\
& x_{M-}^{k+1}=c_{21}^{k+1} x_{M+}^{k}+c_{22}^{k+1} x_{M-}^{k}+c_{23}^{k+1},
\end{aligned}
$$

where

$$
\begin{aligned}
& c_{11}^{k+1}=\frac{a_{22}^{k+1}}{\operatorname{det}} \quad c_{12}^{k+1}=-\frac{a_{12}^{k+1}}{\operatorname{det}} \quad c_{13}^{k+1}=\frac{1}{\operatorname{det}}\left(a_{22}^{k+1} a_{13}^{k+1}-a_{12}^{k+1} a_{23}^{k+1}\right) \\
& c_{21}^{k+1}=-\frac{a_{21}^{k+1}}{\operatorname{det}} \quad c_{22}^{k+1}=\frac{a_{11}^{k+1}}{\operatorname{det}} \quad c_{23}^{k+1}=\frac{1}{\operatorname{det}}\left(a_{11}^{k+1} a_{23}^{k+1}-a_{13}^{k+1} a_{21}^{k+1}\right) \\
& \operatorname{det}=a_{11}^{k+1} a_{22}^{k+1}-a_{12}^{k+1} a_{21}^{k+1} \text {. }
\end{aligned}
$$


The computational efficiency of the model can be improved by storing 4-D arrays $\mathcal{P}_{\alpha \beta}$ of the transition rates evaluated at the quadrature points $\sigma_{R_{i}}$ and $\sigma_{I_{j}}$ and uniformly distributed values of the stress $\sigma^{\ell}$ and temperature $\mathcal{T}^{m}$, where $\sigma_{\min } \leq \sigma^{\ell} \leq \sigma_{\max }$ and $T_{\min } \leq \mathcal{T}^{m} \leq T_{\max }$. For example,

$$
\left[\mathcal{P}_{+A}^{\ell m}\right]_{i j}=p_{+A}\left(\sigma^{\ell}+\sigma_{I_{j}}, \mathcal{T}^{m} ; \sigma_{R_{i}}\right) .
$$

During implementation, the indices $\ell$ and $m$ corresponding to values nearest to $\sigma^{k}$ and $T^{k}$ are found and the $N_{i} \times N_{j}$ matrices $\mathcal{P}_{\alpha \beta}^{\ell m}$ are used to calculate the matrices corresponding to (14), $C_{11}^{k+1}, \cdots, C_{23}^{k+1}$. These matrices can be rapidly calculated using point-wise operations. The matrices of the phase fractions can then be updated in one step using the relations

$$
\begin{gathered}
X_{M+}^{k+1}=C_{11}^{k+1} \cdot \times X_{M+}^{k}+C_{13}^{k+1} \cdot \times X_{M-}^{k}+C_{13}^{k+1} \\
X_{M-}^{k+1}=C_{21}^{k+1} \cdot \times X_{M+}^{k}+C_{23}^{k+1} \cdot \times X_{M-}^{k}+C_{23}^{k+1}, \\
X_{A}^{k+1}=\mathbb{1}-X_{M+}^{k+1}-X_{M-}^{k+1}
\end{gathered}
$$

where point-wise multiplication (.×) and summation are again used. In Equation (15), $\mathbb{1}$ is the $N_{i} \times N_{j}$ identity matrix.

\section{PARAMETER UNCERTAINTY QUANTIFICATION}

Numerous techniques exist to estimate uncertainty in model parameters. Frequentist approaches involve multiple measurements or samples of experimental data. However, collecting large sets of data can be expensive and time consuming. One method that overcomes these limitations is bootstrapping. ${ }^{24-26}$ Here, we are testing the Bayesian framework by using Markov Chain Monte Carlo (MCMC) algorithms. First, we determine the optimal model parameters using standard least-squares approaches. Then, we use the optimization results to determine the covariance matrix for the MCMC's proposal function.

\subsection{Ordinary Least Squares Fit of Model Parameters}

The optimization algorithm minimizes

$$
F(\vec{p})=\frac{1}{2} \sum_{i=1}^{N}\left(\hat{y}\left(t_{i}\right)-y\left(t_{i} ; \vec{p}\right)\right)^{2},
$$

the sum of squared error between the experimentally measured bending angle $\hat{y}\left(t_{i}\right)$ and the model predicted bending angle $y\left(t_{i} ; \vec{p}\right)=\theta\left(t_{i} ; \vec{p}\right)$ given by Equation (1). A complete list of the model parameters $\vec{p}$ is provided in Table 1. Here, we are including all the SMA model parameters in the optimization routine. Alternatively, one could optimize the SMA model parameters (such as $\alpha_{k, m}$ through $V$ in Table 1) using tensile-test data ${ }^{17}$ and then only fit the heat transfer and bending model parameters using the measured bending angle. Including all the parameters will produce a lower sum of squared error but neglects the coupling between parameters.

\subsection{Markov Chain Monte Carlo Methods}

MCMC algorithms are based on Bayes' rule, which relates the posterior density $\pi(\vec{q} \mid \hat{y})$ to a prior density $\pi_{p r}(\vec{q})$ by the relation

$$
\pi(\vec{q} \mid \hat{y})=\frac{p(\hat{y} \mid \vec{q}) \pi_{p r}(\vec{q})}{\int_{\mathbb{R}^{d}} p(\hat{y} \mid \vec{q}) \pi_{p r}(\vec{q}) d \vec{q}},
$$

where $p(\hat{y} \mid \vec{q})$ is the likelihood of observing $\hat{y}$ given parameters $\vec{q}$. For relatively few parameters, the posterior density can be calculated directly by integrating the denominator in Equation (17). However, in higher-dimension parameter spaces, this approach is infeasible.

MCMC methods avoid the difficult integral in Equation (17) by creating a Markov Chain whose stationary distribution is the posterior density $\pi(\vec{q} \mid \hat{y})$. Various MCMC algorithms exist, including the popular MetropolisHastings algorithm ${ }^{7-9,27}$ and adaptive algorithms. ${ }^{28}$ 
Table 1: Model parameters and estimation techniques.

\begin{tabular}{clc}
\hline \hline Variable & Description & Units \\
\hline$\alpha_{k, m}$ & Relative stress density coefficients & - \\
$\beta_{k}$ & Interaction stress density coefficients & - \\
$E_{A}$ & Elastic modulus of austenite & $\mathrm{GPa}$ \\
$E_{M}$ & Elastic modulus of martensite & $\mathrm{GPa}$ \\
$\sigma_{L}$ & Martensite transition stress at temper- & $\mathrm{MPa}$ \\
& ature $T_{L}$ & \\
$T_{L}$ & Lower transition temperature & $\mathrm{K}$ \\
$\Delta \sigma_{T}$ & Hysteresis loop's temperature depen- & $\mathrm{MPa} / \mathrm{K}$ \\
& dence & \\
$\varepsilon_{T}$ & Maximum recoverable strain & $\%$ \\
$\tau$ & Relaxation time & $\mathrm{s}$ \\
$V$ & Layer volume & $\mathrm{m}^{3}$ \\
$h$ & Convection coefficient & - \\
$\gamma$ & Heat transfer parameter & - \\
$\varepsilon_{P}$ & SMA actuator pre-strain & $\% \mathrm{n}$ \\
$a$ & SMA actuator offset from the neutral & $\mathrm{mm}$ \\
& axis & $\mathrm{mm}$ \\
$L$ & Flexible beam length & $\mathrm{N}-\mathrm{cm}^{2}$ \\
$E I$ & Beam elastic modulus and area moment & \\
& of inertia
\end{tabular}

Here, we are using the Random Walk Metropolis algorithm presented in Solonen. ${ }^{9}$ The algorithm calculates new parameters $\vec{q}^{\text {new }}$ from a proposal distribution $f\left(\cdot \mid \vec{q}^{\text {old }}\right)$. The proposal distribution is taken to be Gaussian; therefore, we need an estimate for the covariance matrix $C$. An initial set of parameters $\vec{q}^{*}$ is determined first using standard least-squares approaches. Using the assumption that the residuals are i.i.d., the covariance matrix is approximately

$$
C=\sigma^{2}\left(J^{T} J\right)^{-1},
$$

where $J$ is the Jacobian of the model error

$$
e\left(t_{i}\right)=\hat{y}\left(t_{i}\right)-\vec{y}\left(t_{i} ; \vec{q}^{*}\right) .
$$

The variance in the model error is estimated by

$$
\sigma^{2}=\frac{S S\left(\vec{q}^{*}\right)}{N-\operatorname{length}(\vec{q})},
$$

where the sum of squared errors is

$$
S S(\vec{q})=\sum_{i=1}^{N}\left(\hat{y}\left(t_{i}\right)-\vec{y}\left(t_{i} ; \vec{q}\right)^{2}\right.
$$

for $N$ experimental data points. After initializing the algorithm with the starting point $\vec{q}^{*}$ and covariance matrix $C$, a new set of parameters is proposed using the random walk relation

$$
\vec{q}^{\text {new }}=\vec{q}^{\text {old }}+R \vec{z},
$$

where $R$ is the Cholesky decomposition of $C$ and $\vec{z}$ is a random vector sampled from the standard normal distribution. 
The new set of parameters is accepted with probability

$$
\alpha=\min \left(1, \frac{\pi\left(\vec{q}^{\text {new }} \mid \hat{y}\right) f\left(\vec{q}^{\text {old }} \mid \vec{q}^{\text {new }}\right)}{\pi\left(\vec{q}^{\text {old }} \mid \hat{y}\right) f\left(\vec{q}^{\text {new }} \mid \vec{q}^{\text {old }}\right)}\right) .
$$

Since the Gaussian yields a symmetric proposal $f\left(\vec{q}^{\text {old }} \mid \vec{q}^{\text {new }}\right)=f\left(\vec{q}^{\text {new }} \mid \vec{q}^{\text {old }}\right)$, the ratio in Equation (19) reduces to

$$
\frac{\pi\left(\vec{q}^{\text {new }} \mid \hat{y}\right)}{\pi\left(\vec{q}^{\text {old }} \mid \hat{y}\right)}=\frac{p\left(\hat{y} \mid \vec{q}^{\text {new }}\right) \pi_{p r}\left(\vec{q}^{\text {new }}\right)}{p\left(\hat{y} \mid \vec{q}^{\text {old }}\right) \pi_{p r}\left(\vec{q}^{\text {old }}\right)} .
$$

Note that in Equation (20), the constant $\int_{\mathbb{R}^{d}} p(\hat{y} \mid \vec{q}) \pi_{p r}(\vec{q}) d \vec{q}$ cancels and we know the posterior density up to this normalization constant. Finally, the assumption of a uniform (or uninformative) prior

$$
\pi_{p r}\left(\vec{q}^{\text {old }}\right)=\pi_{p r}\left(\vec{q}^{\text {new }}\right)=1
$$

yields the acceptance ratio

$$
\alpha=\min \left(1, \frac{p\left(\hat{y} \mid \vec{q}^{\text {new }}\right)}{p\left(\hat{y} \mid \vec{q}^{\text {old }}\right)}\right)
$$

Here, the likelihood is given by

$$
p(\hat{y} \mid \vec{q})=\exp \left(-0.5 \sigma^{2} S S(\vec{q})^{2}\right)
$$

with the assumption of Gaussian model errors. For the MCMC, we are using a subset of the parameters listed in Table 1 and therefore have denoted the MCMC parameters with $\vec{q}$ (versus the optimization parameters $\vec{p}$ ). Since the goal is to use the uncertainty quantification results in robust control algorithms, the heat transfer parameters $h$ and $\gamma$ are included, as the heat transfer dynamics has a strong effect on the response of the SMA-actuated beam. Additionally, the parameters related to the bending model are included: $a, \varepsilon_{P}$, and EI. However, all the parameters Table 1 can be included in the MCMC without affecting the convergence of the Monte Carlo algorithm, since the convergence depends on the number of iterations and not the number of parameters. The Random Walk Metropolis algorithm for the SMA-actuated beam is summarized in Algorithm 1. During the optimization and MCMC steps, all the parameters are scaled so that the magnitudes are on the order of unity.

\section{RESULTS}

\subsection{Ordinary Least Squares Fit of Model Parameters}

A prototype of a SMA-actuated flexible beam was constructed to gather experimental data. The system consists of a $0.127 \mathrm{~mm}$ diameter FLEXINOL SMA actuator (Dynalloy, Inc. Tustin, CA) and a $0.5 \mathrm{~mm}$ diameter superelastic Nitinol beam. The bending angle is measured using a trakSTAR 3D magnetic tracking system (Ascension Technology Corporation, Burlington, VT). Complete details of the experiment are provided in Hannen et al. ${ }^{29}$

An input voltage consisting of a sinusoidal function, ramp input, and step input of different magnitudes is used to collect the experimental data shown in Figure 2(a). This input captures the major loop and various minor loops for the SMA actuator. A comparison between the model output using initial parameter estimates and the experimentally measured bending angle is shown in Figure 2(b). A comparison between the fit model and measured bending angle is shown in Figure 2(c). A complete list of the initial parameter estimates, bounds, and least-squares optimal parameters is summarized in Table 2. The bounds are chosen to keep the parameters within a reasonable range of the parameters found in Crews et al. ${ }^{17}$ or within reasonable ranges based on fabrication tolerances.

\subsection{Markov Chain Monte Carlo}

The MCMC algorithm is parallelized using 8 separate threads. Each thread is initialized with the same starting value (the optimal parameters in Table 2) and run for 50,000 iterations to ensure burn-in. The parameter values for each iteration are shown in Figure 3. The resulting histograms are shown in Figure 4.

The results indicate that the optimal parameters were not obtained during the ordinary least-squares minimization. The MCMC eventually approaches the upper bound on $\gamma(5.0)$ and a convection coefficient $h$ around 


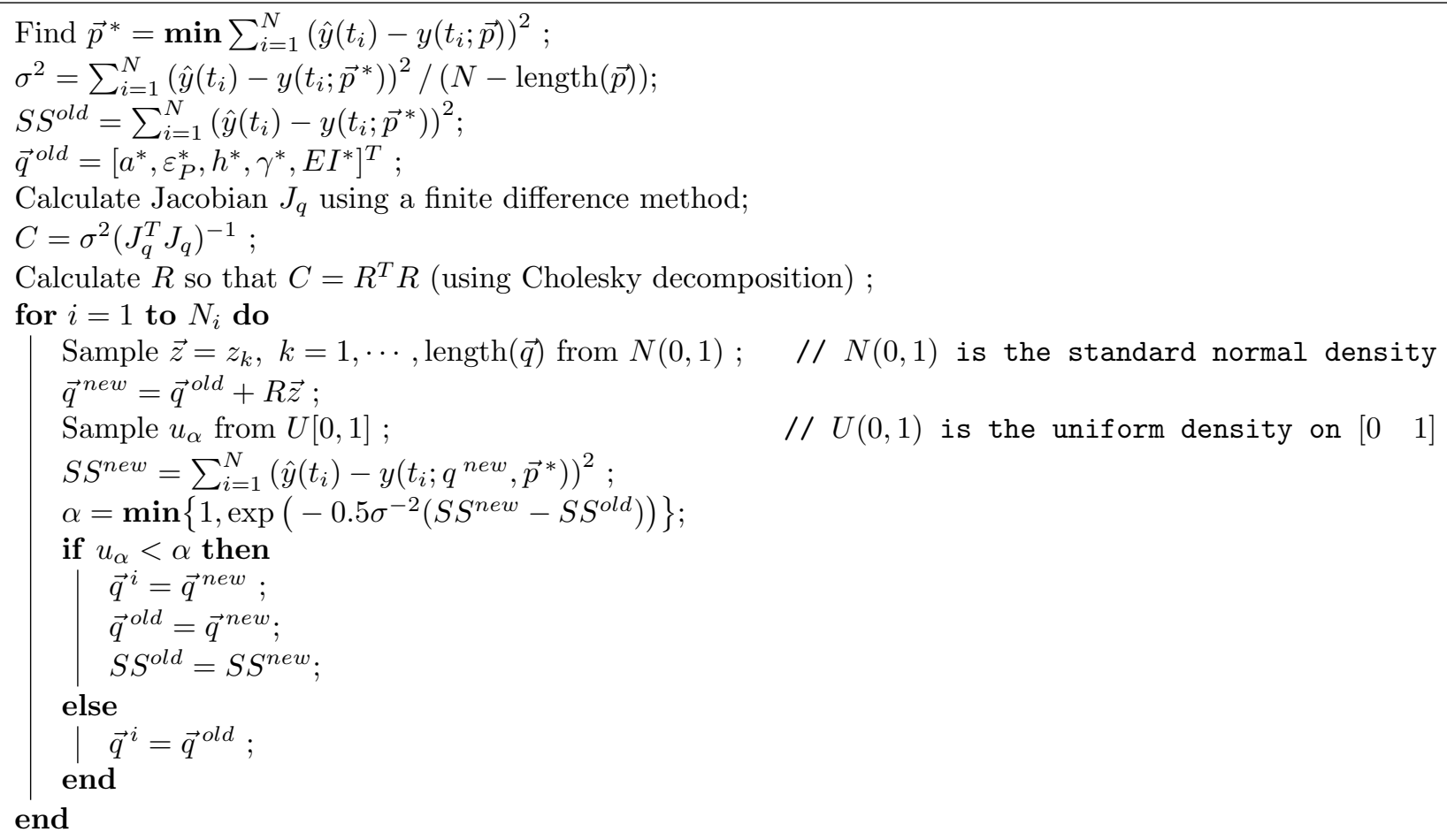

Algorithm 1: Random Walk Metropolis algorithm for the SMA bending actuator.

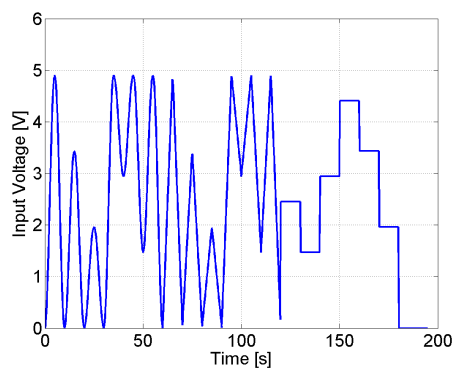

(a)

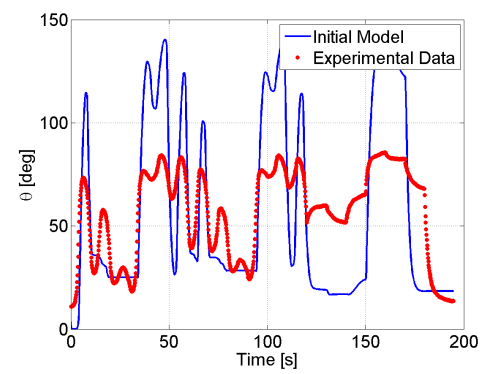

(b)

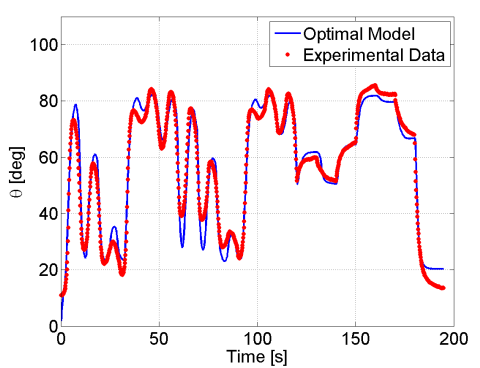

(c)

Figure 2: (a) Input voltage and bending angle comparison between model and experimental data for (b) initial parameter estimates and (c) optimal parameters.

1.65. The root mean squared error (RMSE) corresponding to these values $(\gamma=5.0$ and $h=1.65)$ is $3.93^{\circ}$, compared to a RMSE of $3.98^{\circ}$ for the OLS parameters listed in Table 2. The histograms of the heat transfer parameters $h$ and $\gamma$ are bimodal because the MCMC eventually finds a new set of parameters with similar error as the least-squares optimal parameters. Further investigations are necessary to determine whether relaxing the bounds is appropriate. For control algorithms, we are mainly concerned with bounds on the parameters. The $95 \%$ credible intervals for all the parameters are listed in Table 3.

\section{CONCLUSION}

In this paper, we employed a model for a flexible beam actuated by a single SMA tendon. Additionally, we investigated a systematic approach to quantifying parametric uncertainty using a Markov Chain Monte Carlo algorithm. The results indicate that the posterior densities (a measure of model uncertainty) appear to converge, but additional simulations may be necessary in order to guarantee convergence. Additionally, even though each 
Table 2: Initial optimization values and results.

\begin{tabular}{cccccc}
\hline \hline Variable & $\begin{array}{c}\text { Lower } \\
\text { bound }\end{array}$ & $\begin{array}{c}\text { Upper } \\
\text { bound }\end{array}$ & $\begin{array}{c}\text { Initial } \\
\text { estimate }\end{array}$ & $\begin{array}{c}\text { Optimal } \\
\text { value }\end{array}$ & Units \\
\hline$E_{A}$ & 20.0 & 90.0 & 30.7 & 20.0 & $\mathrm{GPa}$ \\
$E_{M}$ & 20.0 & 50.0 & 26.0 & 50.0 & $\mathrm{GPa}$ \\
$\sigma_{L}$ & 200 & 350 & 295 & 350 & $\mathrm{MPa}$ \\
$\Delta \sigma_{T}$ & 7.0 & 12.0 & 9.2 & 7.04 & $\mathrm{MPa} / \mathrm{K}$ \\
$\varepsilon_{T}$ & 3.5 & 6.0 & 4.36 & 5.11 & $\%$ \\
$\tau$ & 0.001 & 2.0 & 1.81 & 1.2275 & $\mathrm{~s}$ \\
$V$ & $1 \times 10^{-28}$ & $1 \times 10^{-23}$ & $1.0 \times 10^{-25}$ & $7.5 \times 10^{-24}$ & $\mathrm{~m}^{3}$ \\
$h$ & 0.1 & 3.0 & 0.581 & 1.418 & - \\
$\gamma$ & 0.5 & 5.0 & 2.50 & 4.62 & - \\
$\varepsilon_{P}$ & 1.0 & 5.0 & 4.0 & 3.43 & $\%$ \\
$a$ & 0.1 & 3.0 & 1.0 & 2.0 & $\mathrm{~mm}$ \\
$L$ & 60 & 120 & 88 & 106 & $\mathrm{~mm}$ \\
$E I$ & 0.1 & 5.0 & 1.30 & 0.99 & $\mathrm{~N}-\mathrm{cm}^{2}$ \\
\hline
\end{tabular}

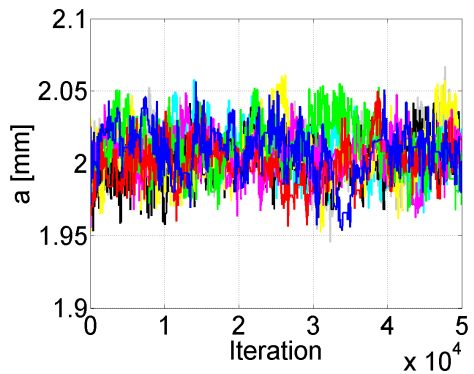

(a)

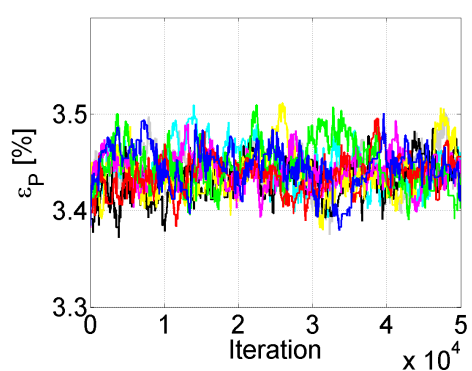

(b)

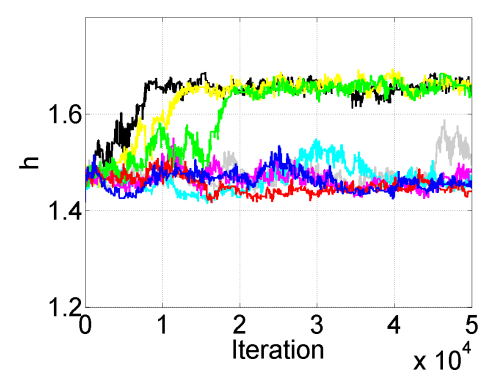

(c)

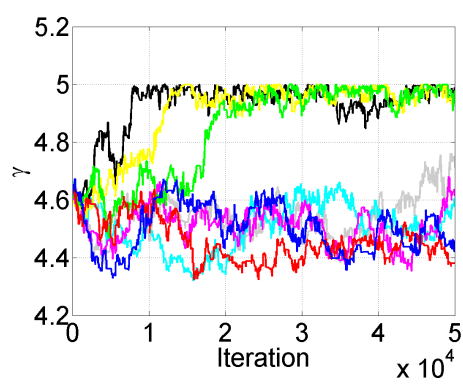

(d)

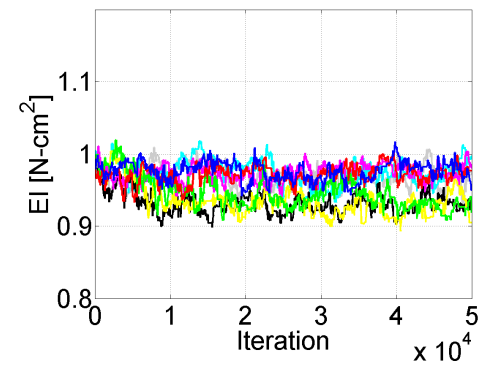

(e)

Figure 3: MCMC threads (different colors) for (a) $a$, (b) $\varepsilon_{P}$, (c) $h$, (d) $\gamma$, and (e) $E I$.

Table 3: Comparison between 95\% credible intervals.

\begin{tabular}{lccccc}
\hline \hline Parameter [units] & $a[\mathrm{~mm}]$ & $\varepsilon_{P}[\%]$ & $h$ & $\gamma$ & $E I\left[\mathrm{~N}-\mathrm{cm}^{2}\right]$ \\
\hline Lower Bound & 1.9746 & 3.4006 & 1.4310 & 4.3674 & 0.9123 \\
Upper Bound & 2.0392 & 3.4871 & 1.6713 & 4.9955 & 0.9976 \\
\hline
\end{tabular}




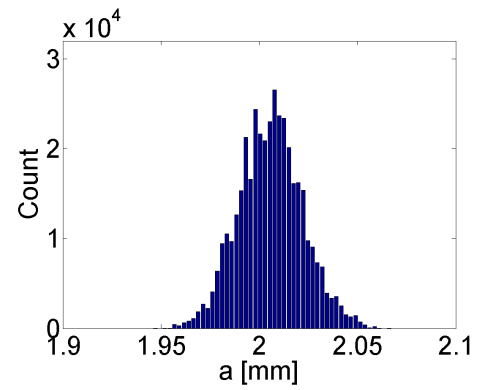

(a)

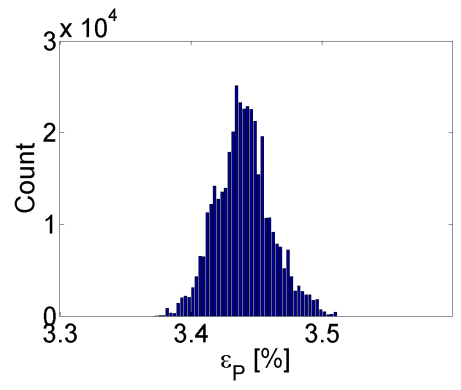

(b)

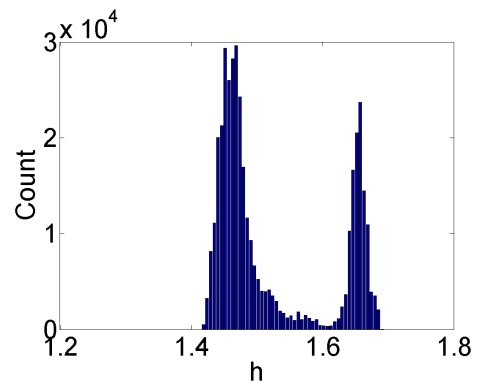

(c)

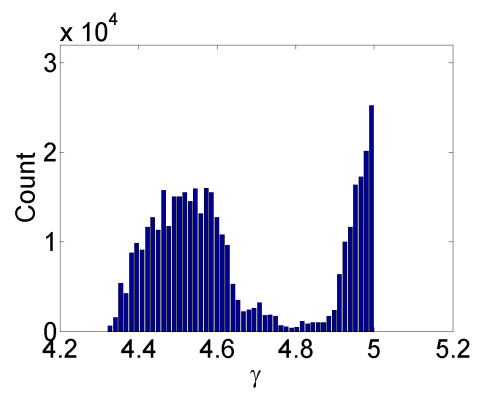

(d)

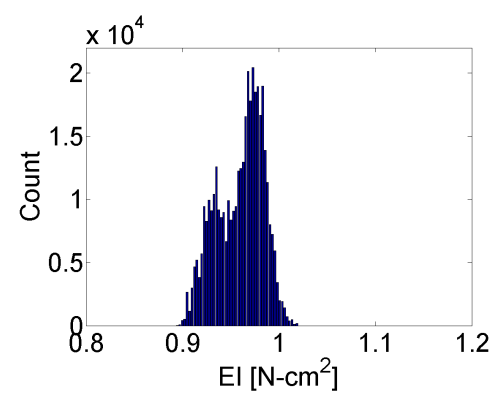

(e)

Figure 4: MCMC histograms for (a) $a$, (b) $\varepsilon_{P}$, (c) $h$, (d) $\gamma$, and (e) $E I$.

parallel thread follows a different path, they are all initialized at the same point. It may advantageous to using different initial values.

Future work will compare different proposal functions and their effect on the convergence of the posterior density. Additionally, MCMC results will be compared to other uncertainty quantification approaches such as bootstrapping. Ultimately, the results will be used in robust control algorithms.

\section{Acknowledgments}

This research was supported in part by the Air Force Office of Scientific Research through the grant AFOSR FA9550-11-10152.

\section{REFERENCES}

1. Veeramani, A., Buckner, G., Owen, S., Bolotin, G., and Cook, R., "Modeling the dynamic behavior of a shape memory alloy actuated catheter," Smart Materials and Structures 19(1), 1-14 (2008).

2. Crews, J. and Buckner, G., "Design optimization of a shape memory alloy actuated robotic catheter," Journal of Intelligent Material Systems and Structures (2011).

3. Laurentis, K. D. and Mavroidis, C., "Mechanical design of a shape memory alloy actuated prosthetic hand," Technology and Health Care 10, 91-106 (2002).

4. Hartl, D., Lagoudas, D., Calkins, F., and Mabe, J., "Use of a ni60ti shape memory alloy for active jet engine chevron application: I. thermomechanical characterization," Smart Materials and Structures 19, 1-14 (2010).

5. Hartl, D., Lagoudas, D., Calkins, F., and Mabe, J., "Use of a ni60ti shape memory alloy for active jet engine chevron application: II. experimentally validated numerical analysis," Smart Materials and Structures 19, 1-14 (2010).

6. Garner, L., Wilson, L., Lagoudas, D., and Rediniotis, O., "Development of a shape memory alloy actuated biomimetic vechicle," Smart Materials and Structures 9, 673-683 (2000). 
7. Kaipio, J. and Somersalo, E., [Statistical and computational inverse problems], vol. 160, Springer Verlag (2005).

8. Gelman, A., [Bayesian data analysis], CRC press (2004).

9. Solonen, A., Monte Carlo methods in parameter estimation of nonlinear models, Master's thesis, Lappeenrante University of Technology (2006).

10. Khalil, H., [Nonlinear Systems], Prentice Hall (2002).

11. Smith, R., [Smart Material Systems: Model Development], SIAM, Philadelphia (2005).

12. Smith, R., Seelecke, S., Dapino, M., and Ounaies, Z., "A unified framework for modeling hysteresis in ferroic materials," Journal of Mechanics and Physics of Solids 54, 46-85 (2005).

13. Smith, R., Dapino, M., Braun, T., and Mortensen, A., "A homogenized energy framework for ferromagnetic hysteresis," IEEE Transactions on Magnetics 42(7) (2006).

14. Smith, R., Seelecke, S., Ounaies, Z., and Smith, J., "A free energy model for hysteresis in ferroelectric materials," Journal of Intelligent Material Systems and Structures 14(11), 719-739 (2003).

15. Smith, R., Hatch, A., Mukherjee, B., and Liu, S., "A homogenized energy model for hysteresis in ferroelectric materials: General density formulation," Journal of Intelligent Material Systems and Structures 16, 713-732 (2005).

16. Smith, R., Seelecke, S., Dapino, M., and Ounaies, Z., "A unified framework for modeling in hystersis in ferroic materials," Journal of the Mechanics and Physics of Solids 54, 46-85 (2005).

17. Crews, J., Smith, R., Pender, K., Hannen, J., and Buckner, G., "Data-driven estimation of the homogenized energy model parameters for shape memory alloys," Journal of Intelligent Material Systems and Structures (2011). Submitted.

18. Hu, Z., Smith, R., and Ernstberger, J., "The homogenized energy model (HEM) for characterizing polarization and strains in hysteretic ferroelectric materials: implementation algorithms and data-driver parameter estimation techniques," Journal of Intelligent Material Systems and Structures (2011). Submitted.

19. Hu, Z., Smith, R., and Ernstberger, J., "Data driven techniques to estimate parameters in a rate-dependent ferromagnetic hysteresis model," Physica:B. To appear.

20. Hu, Z., Smith, R., Stuebner, M., Hay, M., and Oates, W., "Statistical parameter estimation for macro fiber composite actuators using the homogenized energy model," 797806, Proceedings of the SPIE 7978 (2011).

21. Furst, S., Hangekar, R., and Seelecke, S., "Practical implementation of resistance feedback measurement for position control of a flexible smart inhaler nozzle," in [ASME 2010 Conference on Smart Materials, Adaptive Structures, and Intelligent Systems], (2010).

22. Furst, S. and Seelecke, S., "Experimental validation of different methods for controlling a flexible nozzle using embedded sma wires as both positioning actuator and sensor," 79781K, Proceedings of the SPIE 7978 (2011).

23. Crews, J., Development of a shape memory alloy actuated robotic catheter for endocardial ablation: modeling, design optimization, and control, PhD thesis, North Carolina State University (2011).

24. Cao, R., "An overview of bootstrap methods for estimating and predicting in time series," Sociedad de Estadistic e Investagacion Operative Test 8, 95-116 (1999).

25. Zoubir, A. and Iskander, D., "Boostrap methods and applications," IEEE Signal Processing Magazine 24, 10-19 (2007).

26. Liu, R., "Bootstrap procedures under some non-i.i.d. models," The Annals of Statistics 16, 1696-1708 (1988).

27. Chib, S. and Greenberg, E., "Understanding the metropolis-hastings algorithm," American Statistician , 327-335 (1995).

28. Andrieu, C. and Thoms, J., "A tutorial on adaptive mcmc," Statistics and Computing 18(4), 343-373 (2008).

29. Hannen, J., Crews, J., and Buckner, G., "Indirect intelligent sliding mode control of a shape memory alloy actuated flexible beam using hysteretic recurrent neural networks," Smart Materials and Structures (2011). Submitted. 